\title{
A new application of almost increasing sequences
}

\author{
Hikmet Seyhan Özarslan
}




\title{
A NEW APPLICATION OF ALMOST INCREASING SEQUENCES
}

\author{
HIKMET SEYHAN ÖZARSLAN
}

Received June 30, 2011

\begin{abstract}
In the present paper we have proved a more general theorem dealing with $\left|A, p_{n}\right|_{k}$ summability by using almost increasing sequence. This theorem also includes several known results.
\end{abstract}

2000 Mathematics Subject Classification: 40D15; 40F05; 40G99

Keywords: summability factors, absolute matrix summability, almost increasing sequence, infinite series

\section{INTRODUCTION}

A positive sequence $\left(b_{n}\right)$ is said to be almost increasing if there exists a positive increasing sequence $\left(c_{n}\right)$ and two positive constants $\mathrm{A}$ and $\mathrm{B}$ such that $A c_{n} \leq b_{n} \leq$ $B c_{n}$ (see [1]). Let $\sum a_{n}$ be a given infinite series with partial sums $\left(s_{n}\right)$, and let $A=$ $\left(a_{n v}\right)$ be a normal matrix, i.e., a lower triangular matrix of nonzero diagonal entries. Then $A$ defines the sequence-to-sequence transformation, mapping the sequence $s=$ $\left(s_{n}\right)$ to $A s=\left(A_{n}(s)\right)$, where

$$
A_{n}(s)=\sum_{v=0}^{n} a_{n v} s_{v}, \quad n=0,1, \ldots
$$

The series $\sum a_{n}$ is said to be summable $|A|_{k}, k \geq 1$, if (see [9])

$$
\sum_{n=1}^{\infty} n^{k-1}\left|\bar{\Delta} A_{n}(s)\right|^{k}<\infty
$$

where

$$
\bar{\Delta} A_{n}(s)=A_{n}(s)-A_{n-1}(s) .
$$

Let $\left(p_{n}\right)$ be a sequence of positive numbers such that

$$
P_{n}=\sum_{v=0}^{n} p_{v} \rightarrow \infty \quad \text { as } \quad n \rightarrow \infty, \quad\left(P_{-i}=p_{-i}=0, i \geq 1\right)
$$


The sequence-to-sequence transformation

$$
\sigma_{n}=\frac{1}{P_{n}} \sum_{v=0}^{n} p_{v} s_{v}
$$

defines the sequence $\left(\sigma_{n}\right)$ of the Riesz mean or simply the $\left(\bar{N}, p_{n}\right)$ mean of the sequence $\left(s_{n}\right)$, generated by the sequence of coefficients $\left(p_{n}\right)$ (see [6]). The series $\sum a_{n}$ is said to be summable $\left|\bar{N}, p_{n}\right|_{k}, k \geq 1$, if (see [2])

$$
\sum_{n=1}^{\infty}\left(P_{n} / p_{n}\right)^{k-1}\left|\Delta \sigma_{n-1}\right|^{k}<\infty,
$$

and it is said to be summable $\left|A, p_{n}\right|_{k}, k \geq 1$, if (see [8])

$$
\sum_{n=1}^{\infty}\left(\frac{P_{n}}{p_{n}}\right)^{k-1}\left|\bar{\Delta} A_{n}(s)\right|^{k}<\infty,
$$

where

$$
\bar{\Delta} A_{n}(s)=A_{n}(s)-A_{n-1}(s) .
$$

In the special case when $p_{n}=1$ for all $n,\left|A, p_{n}\right|_{k}$ summability is the same as $|A|_{k}$ summability. Also if we take $a_{n v}=\frac{p_{v}}{P_{n}}$, then $\left|A, p_{n}\right|_{k}$ summability is the same as $\left|\bar{N}, p_{n}\right|_{k}$ summability.

In [5], Bor has proved the following theorem for $\left|\bar{N}, p_{n}\right|_{k}$ summability factors of infinite series.

Theorem 1. Let $\left(X_{n}\right)$ be an almost increasing sequence and let there be sequences $\left(\beta_{n}\right)$ and $\left(\lambda_{n}\right)$ such that

$$
\begin{gathered}
\left|\Delta \lambda_{n}\right| \leq \beta_{n}, \\
\beta_{n} \rightarrow 0 \text { as } \quad n \rightarrow \infty \\
\sum_{n=1}^{\infty} n\left|\Delta \beta_{n}\right| X_{n}<\infty \\
\left|\lambda_{n}\right| X_{n}=O(1) .
\end{gathered}
$$

and

$$
\sum_{v=1}^{n} \frac{\left|t_{v}\right|^{k}}{v}=O\left(X_{n}\right) \quad \text { as } \quad n \rightarrow \infty,
$$

where $\left(t_{n}\right)$ is the $n$-th $(C, 1)$ mean of the sequence $\left(n a_{n}\right)$. Suppose further, the sequence $\left(p_{n}\right)$ is such that

$$
\begin{aligned}
P_{n} & =O\left(n p_{n}\right), \\
P_{n} \Delta p_{n} & =O\left(p_{n} p_{n+1}\right) .
\end{aligned}
$$

Then the series $\sum_{n=1}^{\infty} a_{n} \frac{P_{n} \lambda_{n}}{n p_{n}}$ is summable $\left|\bar{N}, p_{n}\right|_{k}, k \geq 1$. 
Remark 1. It should be noted that, from the hypotheses of Theorem $1,\left(\lambda_{n}\right)$ is bounded and $\Delta \lambda_{n}=O(1 / n)$ (see [3]).

\section{THE MAIN RESULT}

The aim of this paper is to generalize Theorem 1 for absolute matrix summability. Before stating the main theorem we must first introduce some further notations.

Given a normal matrix $A=\left(a_{n v}\right)$, we associate two lower semimatrices $\bar{A}=\left(\bar{a}_{n v}\right)$ and $\hat{A}=\left(\hat{a}_{n v}\right)$ as follows:

$$
\bar{a}_{n v}=\sum_{i=v}^{n} a_{n i}, \quad n, v=0,1, \ldots
$$

and

$$
\hat{a}_{00}=\bar{a}_{00}=a_{00}, \quad \hat{a}_{n v}=\bar{a}_{n v}-\bar{a}_{n-1, v}, \quad n=1,2, \ldots
$$

It may be noted that $\bar{A}$ and $\hat{A}$ are the well-known matrices of series-to-sequence and series-to-series transformations, respectively. Then, we have

$$
A_{n}(s)=\sum_{v=0}^{n} a_{n v} s_{v}=\sum_{v=0}^{n} \bar{a}_{n v} a_{v}
$$

and

$$
\bar{\Delta} A_{n}(s)=\sum_{v=0}^{n} \hat{a}_{n v} a_{v} .
$$

Now, we shall prove the following theorem.

Theorem 2. Let $\left(X_{n}\right)$ be an almost increasing sequence. The conditions (1.7)(1.13) of Theorem 1 and

$$
\sum_{v=1}^{n} \frac{p_{v}}{P_{v}}=O\left(X_{n}\right) \text { as } n \rightarrow \infty,
$$

are satisfied. If $A=\left(a_{n v}\right)$ is a positive normal matrix such that

$$
\begin{aligned}
\bar{a}_{n o} & =1, n=0,1, \ldots, \\
a_{n-1, v} & \geq a_{n v}, \text { for } n \geq v+1, \\
a_{n n} & =O\left(\frac{p_{n}}{P_{n}}\right), \\
\left|\widehat{a}_{n, v+1}\right| & =O\left(v\left|\Delta_{v}\left(\widehat{a}_{n v}\right)\right|\right),
\end{aligned}
$$

then the series $\sum_{n=1}^{\infty} a_{n} \frac{P_{n} \lambda_{n}}{n p_{n}}$ is summable $\left|A, p_{n}\right|_{k}, k \geq 1$.

It should be noted that if we take $a_{n v}=\frac{p_{v}}{P_{n}}$, then we get Theorem 1 .

We need the following lemmas for the proof of our theorem. 
Lemma 1. ([7]) If $\left(X_{n}\right)$ an almost increasing sequence, then under the conditions (1.8)-(1.9) we have that

$$
\begin{aligned}
& n X_{n} \beta_{n}=O(1), \\
& \sum_{n=1}^{\infty} \beta_{n} X_{n}<\infty .
\end{aligned}
$$

Lemma 2 ([4]). If the conditions (1.12) and (1.13) are satisfied, then $\Delta\left(P_{n} / p_{n} n^{2}\right)=$ $O\left(1 / n^{2}\right)$.

Proof of Theorem 2. Let $\left(T_{n}\right)$ denotes A-transform of the series $\sum_{n=1}^{\infty} \frac{a_{n} P_{n} \lambda_{n}}{n p_{n}}$. Then we have by (2.3) and (2.4)

$$
\bar{\Delta} T_{n}=\sum_{v=1}^{n} \hat{a}_{n v} \frac{a_{v} P_{v} \lambda_{v}}{v p_{v}} .
$$

Applying Abel's transformation to this sum, we get that

$$
\begin{aligned}
\bar{\Delta} T_{n}= & \sum_{v=1}^{n} \hat{a}_{n v} \frac{v a_{v} P_{v} \lambda_{v}}{v^{2} p_{v}} \\
= & \sum_{v=1}^{n-1} \Delta_{v}\left(\frac{\hat{a}_{n v} P_{v} \lambda_{v}}{v^{2} p_{v}}\right) \sum_{r=1}^{v} r a_{r}+\frac{\hat{a}_{n n} P_{n} \lambda_{n}}{n^{2} p_{n}} \sum_{r=1}^{n} r a_{r} \\
= & \sum_{v=1}^{n-1} \Delta_{v}\left(\frac{\hat{a}_{n v} P_{v} \lambda_{v}}{v^{2} p_{v}}\right)(v+1) t_{v}+\frac{a_{n n} P_{n} \lambda_{n}}{n^{2} p_{n}}(n+1) t_{n} \\
= & \frac{a_{n n} P_{n} \lambda_{n}}{n^{2} p_{n}}(n+1) t_{n}+\sum_{v=1}^{n-1} \Delta_{v}\left(\hat{a}_{n v}\right) \frac{(v+1)}{v^{2}} \frac{P_{v} \lambda_{v}}{p_{v}} t_{v} \\
& +\sum_{v=1}^{n-1} \frac{\hat{a}_{n, v+1} P_{v}}{p_{v}} \Delta \lambda_{v} t_{v} \frac{(v+1)}{v^{2}}+\sum_{v=1}^{n-1} \hat{a}_{n, v+1} \lambda_{v+1} \Delta\left(\frac{P_{v}}{v^{2} p_{v}}\right) t_{v}(v+1) \\
= & T_{n, 1}+T_{n, 2}+T_{n, 3}+T_{n, 4} .
\end{aligned}
$$

Since

$$
\left|T_{n}(1)+T_{n}(2)+T_{n}(3)+T_{n}(4)\right|^{k} \leq 4^{k}\left(\left|T_{n}(1)\right|^{k}+\left|T_{n}(2)\right|^{k}+\left|T_{n}(3)\right|+\left|T_{n}(4)\right|^{k}\right)
$$

to complete the proof of the theorem it is sufficient to show that

$$
\sum_{n=1}^{\infty}\left(\frac{P_{n}}{p_{n}}\right)^{k-1}\left|T_{n, r}\right|^{k}<\infty, \quad \text { for } \quad r=1,2,3,4
$$


Firstly, by using Abel's transformation, we have that

$$
\begin{aligned}
\sum_{n=1}^{m}\left(\frac{P_{n}}{p_{n}}\right)^{k-1}\left|T_{n, 1}\right|^{k} & =O(1) \sum_{n=1}^{m}\left(\frac{P_{n}}{p_{n}}\right)^{k-1} a_{n n}^{k}\left(\frac{P_{n}}{p_{n}}\right)^{k}\left|\lambda_{n}\right|^{k} \frac{\left|t_{n}\right|^{k}}{n^{k}} \\
& =O(1) \sum_{n=1}^{m}\left|\lambda_{n}\right|^{k-1}\left|\lambda_{n}\right| \frac{\left|t_{n}\right|^{k}}{n} \\
& =O(1) \sum_{n=1}^{m}\left|\lambda_{n}\right|^{\left|t_{n}\right|^{k}} \\
& =O(1) \sum_{n=1}^{m-1} \Delta\left|\lambda_{n}\right| \sum_{v=1}^{n} \frac{\left|t_{v}\right|^{k}}{v}+O(1)\left|\lambda_{m}\right| \sum_{n=1}^{m} \frac{\left|t_{n}\right|^{k}}{n} \\
& =O(1) \sum_{n=1}^{m-1}\left|\Delta \lambda_{n}\right| X_{n}+O(1)\left|\lambda_{m}\right| X_{m} \\
& =O(1) \sum_{n=1}^{m-1} \beta_{n} X_{n}+O(1)\left|\lambda_{m}\right| X_{m}=O(1) \quad \text { as } \quad m \rightarrow \infty
\end{aligned}
$$

by virtue of the hypotheses of the theorem and Lemma 1 .

Now, using the fact that $P_{v}=O\left(v p_{v}\right)$ by (1.12), we have that

$$
\sum_{n=2}^{m+1}\left(\frac{P_{n}}{p_{n}}\right)^{k-1}\left|T_{n, 2}\right|^{k}=O(1) \sum_{n=2}^{m+1}\left(\frac{P_{n}}{p_{n}}\right)^{k-1}\left(\sum_{v=1}^{n-1}\left|\Delta_{v}\left(\hat{a}_{n v}\right)\right|\left|\lambda_{v}\right|\left|t_{v}\right|\right)^{k}
$$

Now, applying Hölder's inequality with indices $k$ and $k^{\prime}$, where $k>1$ and $\frac{1}{k}+\frac{1}{k^{\prime}}=1$, we have that

$$
\begin{aligned}
\sum_{n=2}^{m+1}\left(\frac{P_{n}}{p_{n}}\right)^{k-1}\left|T_{n, 2}\right|^{k}= & O(1) \sum_{n=2}^{m+1}\left(\frac{P_{n}}{p_{n}}\right)^{k-1}\left(\sum_{v=1}^{n-1}\left|\Delta_{v}\left(\hat{a}_{n v}\right)\right|\left|\lambda_{v}\right|^{k}\left|t_{v}\right|^{k}\right) \\
& \times\left(\sum_{v=1}^{n-1}\left|\Delta_{v}\left(\hat{a}_{n v}\right)\right|\right)^{k-1} \\
= & O(1) \sum_{n=2}^{m+1}\left(\frac{P_{n}}{p_{n}}\right)^{k-1} a_{n n}^{k-1}\left(\sum_{v=1}^{n-1}\left|\Delta_{v}\left(\hat{a}_{n v}\right)\right|\left|\lambda_{v}\right|^{k}\left|t_{v}\right|^{k}\right) \\
= & O(1) \sum_{v=1}^{m}\left|\lambda_{v}\right|^{k}\left|t_{v}\right|^{k} \sum_{n=v+1}^{m+1}\left|\Delta_{v}\left(\hat{a}_{n v}\right)\right| \\
= & O(1) \sum_{v=1}^{m}\left|\lambda_{v}\right|\left|t_{v}\right|^{k} a_{v v}=O(1) \sum_{v=1}^{m}\left|\lambda_{v}\right|\left|t_{v}\right|^{k} \frac{p_{v}}{P_{v}}
\end{aligned}
$$




$$
\begin{aligned}
& =O(1) \sum_{v=1}^{m-1} \Delta\left|\lambda_{v}\right| \sum_{r=1}^{v} \frac{p_{r}}{P_{r}}\left|t_{r}\right|^{k}+O(1)\left|\lambda_{m}\right| \sum_{v=1}^{m} \frac{p_{v}}{P_{v}}\left|t_{v}\right|^{k} \\
& =O(1) \sum_{v=1}^{m-1}\left|\Delta \lambda_{v}\right| X_{v}+O(1)\left|\lambda_{m}\right| X_{m} \\
& =O(1) \sum_{v=1}^{m-1} \beta_{v} X_{v}+O(1)\left|\lambda_{m}\right| X_{m} \\
& =O(1) \quad \text { as } \quad m \rightarrow \infty
\end{aligned}
$$

by virtue of the hypotheses of the theorem and Lemma 1 .

Now, using Hölder's inequality we have that

$$
\begin{aligned}
& \sum_{n=2}^{m+1}\left(\frac{P_{n}}{p_{n}}\right)^{k-1}\left|T_{n, 3}\right|^{k} \\
& =O(1) \sum_{n=2}^{m+1}\left(\frac{P_{n}}{p_{n}}\right)^{k-1}\left(\sum_{v=1}^{n-1}\left|\hat{a}_{n, v+1}\right|\left|\Delta \lambda_{v}\right|\left|t_{v}\right|\right)^{k} \\
& =O(1) \sum_{n=2}^{m+1}\left(\frac{P_{n}}{p_{n}}\right)^{k-1}\left(\sum_{v=1}^{n-1}\left|\hat{a}_{n, v+1}\right| \beta_{v}\left|t_{v}\right|^{k}\right) \times\left(\sum_{v=1}^{n-1}\left|\hat{a}_{v, v+1}\right| \beta_{v}\right)^{k-1} \\
& =O(1) \sum_{n=2}^{m+1}\left(\frac{P_{n}}{p_{n}}\right)^{k-1} a_{n n}^{k-1}\left(\sum_{v=1}^{n-1}\left|\hat{a}_{n, v+1}\right| \beta_{v}\left|t_{v}\right|^{k}\right)^{m=1} \sum_{v=1}^{m} v \beta_{v} \frac{1}{v}\left|t_{v}\right|^{k} \\
& =\left.O(1) \sum_{n=v+1}^{m} \beta_{v}\left|t_{v} \sum^{m+1}\right| \hat{a}_{n, v+1}\left|=O(1) \sum_{v=1}^{m-1} \Delta\left(v \beta_{v}\right) \sum_{r=1}^{v} \frac{1}{r}\right| t_{r}\right|^{k}+O(1) m \beta_{m} \sum_{v=1}^{m} \frac{1}{v}\left|t_{v}\right|^{k} \\
& =O(1) \sum_{v=1}^{m-1}\left|\Delta\left(v \beta_{v}\right)\right| X_{v}+O(1) m \beta_{m} X_{m} \\
& =O(1) \sum_{v=1}^{m-1} v\left|\Delta \beta_{v}\right| X_{v}+O(1) \sum_{v=1}^{m-1} \beta_{v+1} X_{v+1}+O(1) m \beta_{m} X_{m} \\
& =O(1) \sum^{m} m \rightarrow \infty,
\end{aligned}
$$

by virtue of the hypotheses of the theorem and Lemma 1 . 
Finally, since $\Delta\left(\frac{P_{v}}{v^{2} p_{v}}\right)=O\left(\frac{1}{v^{2}}\right)$, as in $T_{n, 1}$ we have that

$$
\begin{aligned}
& \sum_{n=2}^{m+1}\left(\frac{P_{n}}{p_{n}}\right)^{k-1}\left|T_{n}(4)\right|^{k} \\
& =O(1) \sum_{n=2}^{m+1}\left(\frac{P_{n}}{p_{n}}\right)^{k-1}\left(\sum_{v=1}^{n-1}\left|\hat{a}_{n, v+1}\right|\left|\lambda_{v+1}\right| \frac{\left|t_{v}\right|}{v}\right)^{k} \\
& =O(1) \sum_{n=2}^{m+1}\left(\frac{P_{n}}{p_{n}}\right)^{k-1}\left(\sum_{v=1}^{n-1}\left|\hat{a}_{n, v+1}\right|\left|\lambda_{v+1}\right|^{k} \frac{\left|t_{v}\right|^{k}}{v}\right)\left(\sum_{v=1}^{n-1}\left|\hat{a}_{n, v+1}\right| \frac{1}{v}\right)^{k-1} \\
& =O(1) \sum_{n=2}^{m+1}\left(\frac{P_{n}}{p_{n}}\right)^{k-1}\left(\sum_{v=1}^{n-1}\left|\hat{a}_{n, v+1}\right|\left|\lambda_{v+1}\right|^{k} \frac{\left|t_{v}\right|^{k}}{v}\right)\left(\sum_{v=1}^{n-1}\left|\Delta_{v} \hat{a}_{n v}\right|\right)^{k-1} \\
& =O(1) \sum_{n=2}^{m+1}\left(\frac{P_{n}}{p_{n}}\right)^{k-1} a_{n n}^{k-1}\left(\sum_{v=1}^{n-1}\left|\Delta_{v} \hat{a}_{n v}\right|\left|\lambda_{v+1}\right|^{k} \frac{\left|t_{v}\right|^{k}}{v}\right) \\
& =O(1) \sum_{v=1}^{m} \frac{\left|\lambda_{v+1}\right|}{v}\left|t_{v}\right|^{k} \sum_{n=v+1}^{m+1}\left|\hat{a}_{n, v+1}\right| \\
& =O(1) \sum_{v=1}^{m} \frac{\left|\lambda_{v+1}\right|}{v}\left|t_{v}\right|^{k}=O(1), \quad \text { as } \quad m \rightarrow \infty
\end{aligned}
$$

Therefore we get

$$
\sum_{n=1}^{m}\left(\frac{P_{n}}{p_{n}}\right)^{k-1}\left|T_{n, r}\right|^{k}=O(1) \quad \text { as } \quad m \rightarrow \infty, \quad \text { for } \quad r=1,2,3,4 .
$$

This completes the proof of Theorem 2.

\section{REFERENCES}

[1] N. K. Bari and S. B. Stechkin, "Best approximation and differential properties of two conjugate functions," Tr. Mosk. Mat. Obshch., vol. 5, pp. 483-522, 1956.

[2] H. Bor, "On two summability methods," Math. Proc. Camb. Philos. Soc., vol. 97, pp. 147-149, 1985.

[3] H. Bor, "A note on $\left|\overline{\mathcal{N}}, p_{n}\right|_{k}$ summability factors of infinite series," Indian J. Pure Appl. Math., vol. 18, pp. 330-336, 1987.

[4] H. Bor, "Absolute summability factors for infinite series," Indian J. Pure Appl. Math., vol. 19, no. 7, pp. 664-671, 1988.

[5] H. Bor, "A note on absolute Riesz summability factors," Math. Inequal. Appl., vol. 10, no. 3, pp. 619-625, 2007.

[6] G. H. Hardy, Divergent series. Oxford: At the Clarendon Press (Geoffrey Cumberlege), 1949. 
[7] S. M. Mazhar, “A note on absolute summability factors," Bull. Inst. Math., Acad. Sin., vol. 25, no. 3, pp. 233-242, 1997.

[8] W. T. Sulaiman, "Inclusion theorems for absolute matrix summability methods of an infinite series. IV.” Indian J. Pure Appl. Math., vol. 34, no. 11, pp. 1547-1557, 2003.

[9] N. Tanovic-Miller, “On strong summability," Glas. Mat., III. Ser., vol. 14(34), pp. 87-97, 1979.

\section{Author's address}

\section{Hikmet Seyhan Özarslan}

Department of Mathematics, Erciyes University, 38039 Kayseri, TURKEY

E-mail address: seyhanderciyes.edu.tr; hseyhan38@gmail.com 